Rev. Elev. Méd. vét. Pays trop., 1976, 29 (4) : 353-366.

\title{
Perspectives sur l'alimentation rationnelle des veaux au Sénégal et en zone sahélienne
}

\author{
par FAVRE (B.) (类) et CALVET (H.) (*)
}

\begin{abstract}
RÉSUMÉ
Après les récentes années de sécheresse, la reconstitution de l'élevage sahélien devrait s'orienter vers un mode d'exploitation plus intensif.

Une des formules à envisager consiste au repli précoce des veaux hors de la zone sahélienne vers des régions offrant une écologie et des conditions d'alimentation meilleures. Là, les jeunes animaux subiraient un "réélevage " leur permettant d'atteindre rapidement leur poids d'exploitation.

Une expérimentation de ce type a été poursuivle pendant 9 mois à Sangalkam, ferme située à une quarantaine de kilomètres au Nord de Dakar, suivant plusieurs modalités alimentaires. Les résultats techniques et économiques en sont présentés et discutés par les auteurs.
\end{abstract}

\section{INTRODUCTION}

Le cycle de sécheresse que vient de subir la zone sahélienne a entraîné des pertes de bétail plus ou moins graves suivant les régions.

Il en est résulté que le troupeau sahélien longtemps capable d'assurer l'approvisionnement en viande des grandes cités africaines de la côte ne semble plus pouvoir jouer ce rôle durant les prochaines années. En définitive, cet événement climatique a révélé de façon brutale des faits pressentis par certains, à savoir l'extrême vulnérabilité, dans sa forme actuelle, du troupeau sahélien alors que, parallèlement, la demande en viande croît régulèrement chaque année.

L'élevage de cueillette, forme d'exploitation encore la plus courante dans cette zone, est donc apparu brusquement comme inadapté aux conditions économiques modernes. L'indispensable reconstitution du troupeau ne devrait donc pas se concevoir comme un retour à

(*) Laboratoire national de l'Elevage et de Recherches vétérinaires, B. P. 2057, Dakar-Hann, Sénégal.

(**) Adresse actuelle: INRA, Laboratoire de Pharmacologie, 180, chemin de Tournefeuille, 31300 Toulouse, France. l'état antérieur mais comme une évolution radicale vers un mode plus intensif capable de produire davantage dans une continuité mieux préservée des aléas climatiques.

Les diverses voies d'intensification de la production au niveau des élevages sahéliens sont encore, en général, mal définies. Une de celles qui théoriquement pourraient être envisagées consisterait en un "déstockage " rapide des jeunes et en leur réélevage, hors de la zone sahélienne, dans des régions à écologie meilleure ou plus aisément contrôlée.

Il est inutile d'insister sur les avantages d'une telle opération aussi bien pour les jeunes soustraits ainsi aux conditions climatiques excessives et à la malnutrition des saisons sèches que pour les animaux restant sur place, les naisseurs, qui verraient leurs capacités augmenter sous l'effet d'une diminution de la charge des pâturages.

Ce sont les résultats issus d'une expérimentation de réélevage des veaux, élaborée en fonction des considérations précédentes et conduite à la Ferme de Sangalkam située à une quarantaine de kilomètres au nord de Dakar durant l'année 1973 qui constituent l'objet de cette étude. 


\section{EXPÉRIMENTATION}

En février 1973, 63 veaux mâles zébus Gobra âgés approximativement de 6 à 7 mois et pesant en moyenne $63 \mathrm{~kg} \pm 3$ sont achetés dans la région de Thiès. Leur état général est très médiocre en raison de l'extrême pauvreté des pâturages consécutive à une saison des pluies 1972 presque inexistante.

Dès leur arrivée à Sangalkam, ces animaux subissent les traitements habituels : déparasitage (Tétramisole et quinacrine), vaccinations contre la peste et la péripneumonie.

Ils sont ensuite répartis en 3 lots $\left(n^{\text {os }} 1,2\right.$ et 4) de 20 individus auxquels on adjoint un quatrième lot $\left(\mathrm{n}^{\circ} 3\right)$ composé de 13 veaux métis (Gobra $\times$ pakistanais) en provenance du Centre de Recherches zootechniques de Dara. Ce dernier lot est très hétérogène puisque le poids des individus d'âges différents y varie de 70 à $160 \mathrm{~kg}$.

Les modalités d'alimentation, particulières à chaque lot, présentent cependant un facteur commun : le même concentré est distribué à tous. Sa composition fait l'objet du tableau $\mathrm{n}^{0} \mathrm{I}$.

TABLEAU $N^{\circ} 1$

Composition centésimale du concentré

\begin{tabular}{|c|c|}
\hline Farine de sorgho & 50 \\
\hline Gros son de blé & 42 \\
\hline Tourteau d'arachide .. & 3 \\
\hline Urée. . & 0,5 \\
\hline Polyfos (*) & 1 \\
\hline Carbonate de chaux $\ldots$. & 2,5 \\
\hline Sel & 1 \\
\hline
\end{tabular}

Il est possible d'attribuer à cet aliment les caractéristiques suivantes :

(*) Le polyfos est un phosphate alumino-calcique, produit au Sénégal, titrant au minimum 15 p. 100 de $\mathrm{P}$ et 7 p. 100 de $\mathrm{Ca}$.
— valeur énergétique $=0,8 \mathrm{UF} \mathrm{au} \mathrm{kg}$;

- teneur protéique $=99 \mathrm{MAD} / \mathrm{kg}$;

- rapport $\mathrm{MAD} / \mathrm{UF}=124$;

$-\operatorname{rapport} \mathrm{Ca} / \mathrm{P}=1$;

- prix de revient $=26 \mathrm{~F} \mathrm{CFA} \mathrm{le} \mathrm{kg}$;

- prix de revient d'1 UF $=31 \mathrm{~F}$.

Les lots 1,2 et 3 sont maintenus en parcs, aménagés sur une aire bitumée, équipés de mangeoires fixes et d'abreuvoirs. Le lot 4 est entretenu au pâturage sur une parcelle de Sangalkam ayant une superficie approximative de 50 ha. Le lot 1 reçoit à volonté un aliment composé de 40 p. 100 de coque d'arachide, et de 60 p. 100 de concentré intimement mélangés. Dans les lots 2 et 3 , on distribue un fourrage à volonté, de la fane d'arachide et on rationne, d'autre part, le concentré à raison de $6 \mathrm{~kg}$ par $100 \mathrm{~kg}$ de poids métabolique par animal et par jour (Poids métabolique $=$ Poids vif $\left.\begin{array}{l}0,75 \\ -\mathrm{kg}\end{array}\right)$. Dans le lot 4, la distribution du concentré est effectuée au pâturage et rationnée suivant les mêmes normes.

Les différents composants des rations ont fait l'objet de plusieurs séries d'analyses bromatologiques dont les résultats moyens se trouvent consignés dans le tableau $n^{\circ}$ II.

\section{OBSERVATIONS}

Les principales observations, conduites durant 10 périodes de 4 semaines (281 jours au total) portent sur les points suivants :

- étude de l'évolution pondérale, du gain de poids quotidien (CQM) et de la consommation dans chaque lot ;

- étude des carcasses, après abattage, de certains animaux des lots 2 et 3 .

TABL. $N^{0}$ II-Analyse chimique des divers aliments ( $g$ pour 1000 de Matières sèches (MF)

\begin{tabular}{|c|c|c|c|c|}
\hline Composants & $\begin{array}{l}\text { Mêlange } \\
\text { lot } \pi^{\circ} 1\end{array}$ & Concentrê & $\begin{array}{c}\text { Fane } \\
\text { d'arachide }\end{array}$ & $\begin{array}{l}\text { Pâturages de } \\
\text { saicon sèche }\end{array}$ \\
\hline Matières sẽches & 906 & 894 & 904 & 908 \\
\hline Matières minérales & 49 & 74 & 83 & 124 \\
\hline Matières protéiques & 117 & 162 & 135 & 60 \\
\hline Matières grasses & 37 & 38 & 21 & 13 \\
\hline Matières cellulosiques & 362 & 67 & 345 & 405 \\
\hline Extractif non azote & 435 & 659 & 416 & $39 B$ \\
\hline Phosphore & 6,2 & 11,3 & 2,0 & 1,1 \\
\hline Calcium & 5,3 & 9,9 & 13,3 & 4,6 \\
\hline
\end{tabular}

Rectificatif : Lire dans le titre « Matières fraîches ». 
Durant la première partie de l'expérience, qui a duré 5 mois et demi ( $15 / 2$ au $2 / 8)$, les 4 lots initiaux sont intéressés. A cette date sont pratiqués les abattages et les individus des lots 2 et 3 ayant échappé à cette mesure sont regroupés dans un nouveau lot (lot 2 bis).

En définitive, les données concernant la totalité de l'essai portent sur les lots 1 et 4 demeurés intacts et sur le lot 2 bis artificiellement reconstitué. Les lots 2 et 3 n'ont existé que durant les 5 mois et demi de saison sèche.

Des observations complémentaires, dosages biochimiques et mensurations ont été effectués à différentes périodes de l'expérimentation.

\section{III.1. Données pondérales}

Les poids moyens et les croîts quotidiens moyens (CQM) obtenus au cours de chacune des périodes de 4 semaines font l'objet des tableaux $n^{\circ}$ III et IV.

Les 4 premières semaines correspondent à une période d'adaptation, on observe ensuite un certain nombre de particularités à l'intérieur de chaque lot.

Dans le lot $\mathrm{n}^{\circ} 1$ se produit, au mois d'août, une coupure très nette dans l'évolution pondérale due, en grande partie, à l'apparition d'une avitaminose A manifestée, chez certains individus, par des lésions oculaires caractéristiques.

Une « recharge " en vitamines par voie parentérale rétablit les animaux et rehausse rapidement le croît au niveau antérieur.

Il convient de souligner que cet accident

TABL, N`III-Evolution des poids dens les diffërents lots.

\begin{tabular}{|c|c|c|c|c|c|c|}
\hline \multicolumn{2}{|l|}{ Date des pesées } & $\begin{array}{r}\text { Lot } 1 \\
\text { n } 20 \mathrm{~g}\end{array}$ & $\begin{array}{rl}\text { Lot } & 2 \\
19 & 8\end{array}$ & $\begin{array}{c}\text { Lot } 3 \\
13 \text { métis }\end{array}$ & $\begin{aligned} \text { Lot } 4 \\
19 \mathrm{~g}\end{aligned}$ & $\begin{array}{c}\text { Lot } 2 \text { bis } \\
11 \text { Gobra }+9 \text { mêtis }\end{array}$ \\
\hline \multicolumn{2}{|l|}{$15 / 2$} & 52,3 & 61,6 & 108,1 & 75,3 & 75,1 \\
\hline \multicolumn{2}{|l|}{$15 / 3$} & 66,0 & 72,9 & 123,3 & 85,6 & 86,9 \\
\hline \multicolumn{2}{|l|}{$13 / 4$} & 83,4 & $B B, 7$ & 147,8 & 102,0 & 106,4 \\
\hline \multicolumn{2}{|l|}{$10 / 5$} & 97,8 & 103,4 & 171,9 & 113,8 & 124,1 \\
\hline \multicolumn{2}{|l|}{$7 / 6$} & 119,2 & 120,7 & 197,5 & 126,9 & 144,9 \\
\hline \multicolumn{2}{|l|}{$5 / 7$} & 140,5 & 137,0 & 221,5 & 138,2 & 165,2 \\
\hline \multicolumn{2}{|l|}{$2 / B$} & 162,2 & 157,3 & 247,2 & 156,8 & 188,4 \\
\hline \multicolumn{2}{|l|}{$30 / 8$} & 169,9 & & & 172,1 & 202,3 \\
\hline \multicolumn{2}{|l|}{$27 / 9$} & 176,9 & & & 191,5 & 218,6 \\
\hline \multicolumn{2}{|l|}{$25 / 10$} & 195,9 & & & 210,5 & 241,0 \\
\hline \multicolumn{2}{|l|}{$22 / 11$} & 213,1 & & & 224,9 & 256,4 \\
\hline \multirow{2}{*}{$\begin{array}{l}\text { Droites de régres- } \\
\text { sion des poids en } \\
\text { fonction du temps. }\end{array}$} & Pentes & 16,28 & 15,97 & 23,69 & 15,11 & 18,72 \\
\hline & $\begin{array}{c}\text { Terme } \\
\text { constant }\end{array}$ & 52,86 & 58,01 & 102,82 & 69,66 & 70,87 \\
\hline
\end{tabular}

Poids exprimés en kg.

TABL. N ${ }^{D}$ IV -C.Q.M. moyen dans chaque $\operatorname{lot}(\mathrm{g} / \mathrm{j}$ our $)$

\begin{tabular}{|c|c|c|c|c|c|}
\hline D a f e s & Lot 1 & Lot 2 & Lot 3 & Lot 4 & Lot 2 bis \\
\hline $15 / 2$ & 489 & 396 & 543 & 368 & 421 \\
$15 / 3$ & 597 & 545 & 831 & 566 & 674 \\
$13 / 4$ & 533 & 544 & 830 & 448 & 656 \\
$10 / 5$ & 764 & 618 & 914 & 468 & 742 \\
$7 / 6$ & 761 & 582 & 861 & 404 & 724 \\
$5 / 7$ & 775 & 725 & 918 & 664 & 829 \\
$2 / 8$ & 282 & & & 546 & 597 \\
$30 / 8$ & 250 & & & 689 & 800 \\
$27 / 9$ & 679 & & & 579 & 550 \\
$25 / 10$ & & & & & $647 \pm 95$ \\
$22 / 11$ & & & & $534 \pm 82$ & 516 \\
\hline
\end{tabular}


pathologique ne se rencontre que dans le lot 1 . Dans les autres, la fane d'arachide ou les fourrages naturels semblent suffisamment riches en provitamine A pour protéger les animaux de la carence.

Dans le lot 4, entretenu sur pâturage, les gains de poids durant la saison sèche se maintiennent à un niveau inférieur malgré un apport alimentaire supplémentaire en mai, juin et juillet. En effet, au concentré habituel on ajoute durant ces 3 mois du tourteau d'arachide puis des graines de coton.

Par contre, après les premières pluies et le renouveau des pâturages, la croissance dans ce lot devient comparable à celle des autres.

\section{2. Données concernant l'alimentation}

Les valeurs des principaux critères intéressant la consommation journalière moyenne par individu au cours de chaque période sont rassemblées dans le tableau $V$.

L'examen du tableau $n^{\circ} \mathrm{V}$ permet un certain nombre de constatations :

a) La consommation spontanée de matières sèches, exprimée en $\mathrm{kg}$ de $\mathrm{MS}$ par $100 \mathrm{~kg}$ de poids vif est sensiblement plus élevée chez les jeunes bovins que chez les adultes.

Dans le cadre de l'expérimentation, elle a été en moyenne de $4,3 \mathrm{~kg}$ dans le lot 1 mais a diminué du début à la fin de l'essai.

Au début, les animaux âgés approximativement de 6 mois consomment près de $5 \mathrm{~kg}$ de MS. En novembre et à l'âge de 15 mois, ils n'absorbent plus que $3,5 \mathrm{~kg}$.

b) La consommation en MS dans le lot 1 a été plus élevée que dans les autres. On se rappelle que dans le premier cas l'aliment servi à volonté se compose d'un mélange de coque d'arachide et de concentré. Dans les autres, concentré rationné et fane d'arachide ad libitum sont servis séparément.

c) Dans le lot 1 , à l'époque où elle se produit, la carence en vitamine $A$ a un effet très sensible sur la production et sur l'indice de consommation alors que les quantités consommées demeurent à peu près les mềmes.

d) Pour le lot entretenu au pâturage (lot 4), seules les quantités de concentré distribuées sont connues. Pour tenter d'évaluer l'apport du pâturage, nous avons procédé de la façon suivante :
Les valeurs proposées par JACQUOT et al. (1964, p. 1896 et suivantes) permettent d'évaluer les besoins énergétiques d'entretien $\left(B_{U F}\right)$ pour des bovins en croissance ou en engraissement, par la relation : $B_{U F}=0,0364_{p} 0,75$, où $P$ est le poids vif exprimé en $\mathrm{kg}$.

Les besoins de production pour cette catégorie de bétail et à un âge compris entre 6 et 15 mois peuvent être calculés à partir des performances obtenues dans le lot 1 . On trouve en moyenne 2 UF par $\mathrm{kg}$ de gain.

Ces deux séries de données permettent alors d'établir le tableau VI.

TABL. N*VI-Evaluation de 1 'apport du pâturage.

\begin{tabular}{|c|c|c|c|}
\hline Période & $\begin{array}{c}\text { Besoin des } \\
\text { animaux }\end{array}$ & $\begin{array}{c}\text { Valeur du } \\
\text { concentrề }\end{array}$ & $\begin{array}{c}\text { Apport du } \\
\text { pâtuxage }\end{array}$ \\
\hline 1 & 1,92 & 1,78 & 0,14 \\
2 & 2,43 & 1,66 & 0,77 \\
3 & 2,35 & 1,60 & 0,75 \\
4 & 2,46 & $1,87^{+}$ & 0,59 \\
5 & 2,43 & $2,59^{++}$ & 0,16 \\
6 & 3,07 & $2,61^{++}$ & 0,46 \\
7 & 2,97 & 2,13 & 0,84 \\
8 & 3,38 & 2,30 & 1,08 \\
9 & 3,50 & 2,52 & 0,98 \\
10 & 3,29 & 2,62 & 0,67 \\
\hline
\end{tabular}

Lire : apport énergétique.

L'apport du pâturage au cours de la saison sèche, exprimé en UF par animal et par jour est variable mais demeure faible (1/2 UF en moyenne).

Après les pluies, sa contribution devient plus importante mais éphémère puisque, dès novembre, on se rapproche des valeurs de saison sèche.

\section{3. Résultats des abattages}

Le « réélevage » des veaux doit normalement déboucher sur un certain nombre de spéculations économiques qui sont, en fonction de l'âge des animaux :

- le veau de boucherie tel qu'il se présente aux abattoirs de Dakar,

— l'animal plus âgé de 15 à 18 mois pouvant correspondre à un baby beef de type africain,

- enfin, vers 2 ans, le bœuf de labour ou le géniteur précoce.

Or, après 6 mois de cet essai, un certain nombre d'animaux du lot 2 correspond à la 
TABLEAU $\mathrm{N}^{\circ} \mathrm{V}$ - Consommation journalière.

\begin{tabular}{|c|c|c|c|c|c|c|c|c|c|c|c|c|}
\hline & \multicolumn{3}{|c|}{ Lot 1} & \multicolumn{3}{|c|}{ Lot 2} & \multicolumn{3}{|c|}{ Lot 3} & \multicolumn{3}{|c|}{ Lot $2 \mathrm{bis}$} \\
\hline & $\begin{array}{l}\mathrm{kg} \text { de MS } \\
\text { par } \\
100 \mathrm{~kg} \text { vif }\end{array}$ & $\begin{array}{l}\text { Valeur } \\
\text { UF } \\
\text { ration }\end{array}$ & $\begin{array}{c}\text { Indice } \\
\text { consommation }\end{array}$ & $\begin{array}{c}\mathrm{kg} \text { de MS } \\
\text { par } \\
100 \mathrm{~kg} \text { vif }\end{array}$ & $\begin{array}{c}\text { Valeur } \\
\text { UF } \\
\text { ration }\end{array}$ & $\begin{array}{c}\text { Indice } \\
\text { consommation }\end{array}$ & $\begin{array}{c}\mathrm{kg} \text { de MS } \\
\text { par } \\
100 \mathrm{~kg} \text { vif }\end{array}$ & $\begin{array}{c}\text { Valeur } \\
\text { uF } \\
\text { ration }\end{array}$ & $\begin{array}{l}\text { Indice } \\
\text { consommation }\end{array}$ & $\begin{array}{c}\mathrm{kg} \text { de MS } \\
\text { par } \\
100 \mathrm{~kg} \text { vif }\end{array}$ & $\begin{array}{l}\text { Valeur } \\
\text { UF } \\
\text { ration }\end{array}$ & $\begin{array}{l}\text { Indice } \\
\text { consommation }\end{array}$ \\
\hline 1 & 4,8 & 1,5 & 3,1 & 3,9 & 1,9 & 4,8 & 3,5 & 2,8 & 5,2 & & & \\
\hline 2 & 4,8 & 1,9 & 3,2 & 4,2 & 2,2 & 4,0 & 3,7 & 3,2 & 3,9 & & & \\
\hline 3 & 4,9 & 2,4 & 4,4 & 4,2 & 2,5 & 4,7 & 3,7 & 3,7 & 4,5 & & & \\
\hline 4 & 5,2 & 3,0 & 3,9 & 3,9 & 2,7 & 4,4 & 3,4 & 4,0 & 4,4 & & & \\
\hline 5 & 4,7 & 3,2 & 4,2 & 3,6 & 3,0 & 5,1 & 3,4 & 4,4 & 5,1 & & & \\
\hline 6 & 4,1 & 3,3 & 4,3 & 3,6 & 3,3 & 4,5 & 3,4 & 4,9 & 5,3 & & & \\
\hline 7 & 4,0 & 3,5 & 12,4 & & & & & & & 3,7 & 4,4 & 8,9 \\
\hline 8 & 3,9 & 3,6 & 14,2 & & & & & & & 3,6 & 4,6 & 7,9 \\
\hline 9 & 3,5 & 3,5 & 6,1 & & & & & & & 3,3 & 4,8 & 6,0 \\
\hline 10 & 3,5 & 3,7 & 6,0 & & & & & & & 3,2 & 4,9 & 8,9 \\
\hline \begin{tabular}{l}
\multicolumn{1}{c}{$\bar{x}$} \\
Interval le \\
confiance \\
5 p.100
\end{tabular} & $4,3 \pm 0,4$ & $2,9 \pm 0,5$ & $6,0 \pm 2,8$ & $3,9 \pm 0,3$ & $2,6 \pm 0,5$ & $4,5 \pm 0,4$ & $3,5 \pm 0,1$ & $3,8 \pm 0,8$ & $4,7 \pm 0,5$ & $3,4 \pm 0,3$ & $4,6 \pm 0,3$ & $7,9 \pm 2,2$ \\
\hline
\end{tabular}


TABLEAU $N^{*}$ VII -Veau de boucherie

\begin{tabular}{|c|c|c|c|c|c|c|}
\hline $\mathrm{N}^{\bullet}$ & $\begin{array}{l}\text { Poids } \\
\text { initial en } \mathrm{kg}\end{array}$ & $\begin{array}{l}\text { Poids } \\
\text { final en kg }\end{array}$ & $\begin{array}{c}\mathrm{C} . Q . \mathrm{M}, \\
\mathrm{g} / \mathrm{j}\end{array}$ & $\begin{array}{l}\text { Poids vif } \\
\text { aprēs jeûne }\end{array}$ & $\begin{array}{l}\text { Poids de } \\
\text { carcasse }\end{array}$ & $\begin{array}{l}\text { Rendement } \\
\text { commercial }\end{array}$ \\
\hline 079 & 57,7 & 160,7 & 613 & 157 & 79 & 50,3 \\
\hline 090 & 66,7 & 135,3 & 408 & 124 & 70 & 56,4 \\
\hline 105 & 61,3 & 154,0 & 552 & 152 & 83 & 54,6 \\
\hline 405 & 70,3 & 148,0 & 463 & 143 & 77 & 53,8 \\
\hline 489 & 62,3 & 153,0 & 542 & 145 & 70 & 48,3 \\
\hline 544 & 63,7 & 163,3 & 593 & 159 & 83 & 52,8 \\
\hline 726 & 52,7 & 132,7 & 476 & 128 & 68 & 53,1 \\
\hline 744 & 55,3 & 157.7 & 598 & 152 & 77 & 50,7 \\
\hline 797 & 64,3 & 154,3 & 536 & 150 & 81 & 54,0 \\
\hline 943 & 56.3 & 140,7 & 502 & 140 & 75 & 53,6 \\
\hline 1000 & 61,0 & 146,7 & 510 & 141 & 78 & 55,3 \\
\hline Moyenne & $61,1 \pm 3,5$ & $149,5 \pm 6,7$ & $527 \pm 42$ & $145 \pm 7$ & $76 \pm 4$ & $53,0 \pm 1,6$ \\
\hline
\end{tabular}

TABLEAU N $N^{a}$ VIII -Baby beef du lot 3.

\begin{tabular}{|c|c|c|c|c|c|c|}
\hline $\mathrm{N}^{\circ}$ & $\begin{array}{l}\text { Poids } \\
\text { initial en } \mathrm{kg}\end{array}$ & $\begin{array}{l}\text { Poids } \\
\text { final en } \mathrm{kg}\end{array}$ & $\begin{array}{l}\text { C.Q.M. } \\
\mathrm{g} / \mathrm{j}\end{array}$ & $\begin{array}{l}\text { Poids vif } \\
\text { aprēs jeûne }\end{array}$ & $\begin{array}{l}\text { Poids de } \\
\text { carcasse }\end{array}$ & $\begin{array}{l}\text { Rendement } \\
\text { commercial }\end{array}$ \\
\hline 081 & 110,3 & 265,0 & 921. & 257 & 148 & 57,6 \\
\hline 082 & 113,0 & 281,0 & 1000 & 280 & 156 & 55,7 \\
\hline 085 & 102,0 & 258,7 & 933 & 261 & 145 & 55,6 \\
\hline 142 & 145,0 & 324,7 & 1070 & 319 & 177 & 55,5 \\
\hline 275 & 142,0 & 297,3 & 924 & 283 & 156 & 55,1 \\
\hline 278 & 158,3 & 317,3 & 946 & 318 & 177 & 55,6 \\
\hline $\begin{array}{l}\text { Moyenne } \\
\pm \mathrm{t} 0,05\end{array}$ & $128,4 \pm 24,0$ & $290,7+28,5$ & $966 \pm 62$ & $286 \pm 28$ & $160 \pm 15$ & $55,9 \pm 0,9$ \\
\hline
\end{tabular}

spéculation "veau de boucherie » qui s'adresse dans les conditions du marché local à de jeunes animaux capables de fournir des carcasses de 70 à $80 \mathrm{~kg}$.

Dans le lot 3, par contre, les individus les plus lourds peuvent être assimilés à du baby beef local fournissant de 150 à $170 \mathrm{~kg}$ de carcasse. Les résultats des abattages pratiqués sur ces deux lots sont présentés dans les tableaux VII et VIII.

Les Gobras ont donné des carcasses de poids et de qualité bouchère satisfaisants, avec un rendement relativement faible pour des veaux de 12 mois, après une croissance homogène supérieure à $500 \mathrm{~g} / \mathrm{j}$.

Après avoir extériorisé des potentialités de croissance très intéressantes, puisque le croît quotidien moyen se situe aux environs du $\mathrm{kg}$ par jour, les métis d'environ 15 mois ont donné des carcasses de $160 \mathrm{~kg}$ avec un rendement de 56 p. 100 , ce qui est loin des performances européennes, mais nettement supérieur à celles observées avec les races locales au même âge.

\section{4. Mensurations}

Deux types de mensurations ont été pratiqués au cours de chaque pesée : mesure du périmètre thoracique et largeur scapulo-ischiale.

L'évolution de ces deux séries de mesures fait l'objet des tableaux IX et X.

TABL. $\mathrm{N}^{\mathbb{0}} \mathrm{IX}-$ Evolution du périmètre thoracique

\begin{tabular}{|c|c|c|c|c|}
\hline $\mathrm{N}^{\circ}$ pesée & Lot 1 & Lot 2 & Lot 3 & Lot 4 \\
\hline 1 & 86,9 & 91,8 & - & 95,7 \\
2 & 94,2 & 98,9 & 115,8 & 102,8 \\
3 & 99,5 & 103,4 & 121,3 & 107,0 \\
4 & 106,3 & 109,7 & 127,3 & 110,4 \\
5 & 112,4 & 115,6 & 134,1 & 116,2 \\
6 & 119,4 & 120,3 & 140,4 & 119,1 \\
7 & 125,8 & 126,2 & 144,6 & 124,0 \\
8 & 130,2 & - & - & 128,9 \\
9 & 131,4 & - & - & 132,8 \\
10 & 135,4 & - & - & 138,1 \\
11 & 139,9 & & & 140,3 \\
\hline
\end{tabular}

Périmètre exprimé en $\mathrm{cm}$. 
TABL. $\mathrm{N}^{\circ} \mathrm{X}$-Evolution de la longueur scapulo-ischiale

\begin{tabular}{|c|r|r|r|r|}
\hline$N^{*}$ perie & Lot 1 & Lot 2 & Lot 3 & Lot 4 \\
\hline 1 & 78,3 & 82,7 & - & 88,1 \\
2 & 85,5 & 91,1 & 102,5 & 95,6 \\
3 & 87,6 & 94,3 & 110,6 & 98,8 \\
4 & 96,8 & 100,4 & 116,7 & 104,1 \\
5 & 100,3 & 104,0 & 117,4 & 107,0 \\
6 & 103,4 & 104,0 & 120,7 & 107,5 \\
7 & 108,8 & 110,4 & 125,9 & 112,1 \\
8 & 113,3 & - & - & 118,7 \\
9 & 115,8 & - & - & 122,9 \\
10 & 120,6 & - & - & 125,6 \\
11 & 120,3 & - & - & 125,7 \\
\hline
\end{tabular}

Longueur exprimée en $\mathrm{cm}$.

Les corrélations calculées, d'une part, entre le poids et le périmètre thoracique et, d'autre part, entre le poids et la longueur scapulo-ischiale se sont révélées hautement significatives. Dans le premier cas, le coefficient de corrélation, est de 0,99 ; dans le second cas de 0,97 .
Il est donc possible à un éleveur ne disposant pas d'un pèse-bétail de surveiller l'évolution des poids de ses animaux avec une approximation suffisante, en effectuant ces mesures.

\section{III.5. Dosages biochimiques}

Sept séries de prélèvements ont été effectuées au cours de l'expérimentation, avec la mesure de l'hématocrite, les dosages de l'hémoglobine, des protéines, de l'urée sanguine, du calcium, du potassium, du cuivre, du zinc, des transaminases SGOT et SGPT.

Les résultats moyens en fonction de l'alimentation (lots 2,2 bis, 4) et de la saison sèche (hivernage, posthivernage) font l'objet $\mathrm{du}$ tableau général $n^{\circ}$ XI.

N. B. Lorsqu'une seule valeur est portée dans chaque case c'est que les lots ne sont pas significativement différents. Il s'agit alors de la moyenne des lots.

TABLEAU $N^{*} \mathrm{XI}$ - Rêsul tat to biochimiques

\begin{tabular}{|c|c|c|c|c|c|}
\hline E1éments & Réception & Saison sèche & Hivernage & Post-hivernege & $\mathrm{x}$ \\
\hline Hématocrite & $\begin{array}{l}36,3 \pm 1,6 \\
47,2^{(2)} 4,7\end{array}$ & $37,3 \pm 0,8$ & $36,0 \pm 1,1$ & $33,0 \pm 0,8$ & $35,4 \pm 0,6$ \\
\hline Hémoglobine & $\begin{array}{c}11,0 \pm 0,6 \\
(\overline{2}) \\
14,5 \pm 1,4\end{array}$ & $10,0 \pm 0,3$ & $10,3 \pm 0,5$ & $11,3 \pm 0,4$ & $10,6 \pm 0,2$ \\
\hline Protéines & $64,7 \pm 4,0$ & $\begin{array}{l}68,8 \pm 4,5 \\
52,9^{(2)} 6,0\end{array}$ & $70,1 \pm 2,0$ & $71,2 \pm 2,0$ & $70,0 \pm 1,6$ \\
\hline Urêe & $0,359 \pm 0,022$ & $\begin{array}{l}0,370 \pm 0,02 \\
0,217^{(1)} 0,02\end{array}$ & $\begin{array}{c}0,366 \pm 0,02 \\
(\overline{1}) \\
0,308 \pm 0,02\end{array}$ & $\begin{array}{c}0,402 \pm 0,02 \\
(\overline{1}) \\
0,324 \pm 0,03\end{array}$ & $\begin{array}{c}0,380 \pm 0,011 \\
0,280 \pm 0,017\end{array}$ \\
\hline$P$ & $95,9 \pm 7,8$ & $107,3 \pm 2,5$ & $100,4 \pm 3,4$ & $86,6 \pm 2,0$ & $98,2 \pm 1,9$ \\
\hline $\mathrm{Ca}$ & $104,5 \pm 3,1$ & $109,4 \pm 1,3$ & $95,1 \pm 1,7$ & $\begin{array}{c}97,5 \pm 2,5 \\
(\overline{2}) \\
84,5 \pm 9,2\end{array}$ & $100,9 \pm 1,2$ \\
\hline $\mathrm{Mg}$ & $33,4 \pm 2,0$ & $\begin{array}{c}27,3 \pm 0,7 \\
\left(\frac{3}{)}\right) \\
34,8 \pm 3,2\end{array}$ & $\begin{array}{c}24,8 \pm 0,9 \\
(\overline{3}) \\
32,0 \pm 2,3\end{array}$ & $\begin{array}{c}25,3 \pm 0,8 \\
\left(\frac{3}{3}\right) \\
33,2 \pm 3,8\end{array}$ & $\begin{array}{c}25,8 \pm 0,5 \\
\left(\frac{3}{)}\right) \\
33,3 \pm 1,8\end{array}$ \\
\hline $\mathrm{Na}$ & $3590 \pm 72$ & $3604 \pm 25$ & $3433 \pm 51$ & $3334 \pm 71$ & $3458^{\circ} \pm 33$ \\
\hline $\mathrm{K}$ & $210,1 \pm 6,2$ & $218,3 \pm 3,5$ & $187,6 \pm 4,5$ & $177,7 \pm 4,9$ & $194,8 \pm 3,2$ \\
\hline $\mathrm{Cu}$ & $0,73 \pm 0,11$ & $0,73 \pm 0,03$ & $0,67 \pm 0,03$ & $0,79 \pm 0,03$ & $0,73 \pm 0,02$ \\
\hline $2 n$ & $1,91 \pm 0,11$ & $1,35 \pm 0,04$ & $1,62 \pm 0,0 \mathrm{~B}$ & $1,45 \pm 0,04$ & $1,47 \pm 0,04$ \\
\hline SGOT & & $99,2 \pm 8,7$ & $102,6 \pm 10,7$ & $72,3 \pm 8$ & $91,0 \pm 5,5$ \\
\hline SGPT & & $28,3 \pm 2,2$ & $28,0 \pm 1,3$ & $31,6 \pm 1,2$ & $29,3 \pm 1,1$ \\
\hline
\end{tabular}




\section{MI.6. Manifestations pathologiques}

Malgré le mauvais état des animaux à l'achat, les mortalités se sont limitées à 3 individus qui n'ont $\mathrm{pu}$, au début de l'expérimentation, être récupérés.

La seule pathologie observée s'est produite dans le lot 1 en fin de saison sèche. Due à une carence en vitamine A, elle s'est traduite par des lésions oculaires et une diminution sensible de la croissance.

Le protocole avait prévu l'injection chaque mois, et sur la moitié de l'effectif de chaque lot, de $5 \mathrm{ml}$ d'un complexe vitaminique comprenant pour $100 \mathrm{ml} 50000000$ UI de vitamine A, $20000000 \mathrm{UI}$ de vitamine $D_{3}$ et $5 \mathrm{~g}$ de vitamine E. Or, les troubles oculaires se sont manifestés uniquement sur les individus non traités du lot 1 et la différence entre les gains de poids dans les 2 groupes n'est devenu significative que durant cette période. Après traitement de tout l'effectif le 19 juillet, les troubles s'effacent et la croissance reprend normalement.

Le tableau XII témoigne de ces évolutions

TABL. $N^{\circ} X I I-E f f e t s$ des injections de vitemine sur la croissance du lot 1

\begin{tabular}{|c|c|c|c|}
\hline Période & $\left\{\begin{array}{c}\text { Groupe } \\
\text { traitê }(n-10)\end{array}\right.$ & $\begin{array}{l}\text { Groupe non } \\
\text { traitë }(n=9)\end{array}$ & Valeur de $F$ \\
\hline 1 & $13,8 \pm 3,0$ & $1366 \pm 3,5$ & $0,009 \mathrm{NS}$ \\
\hline 2 & $16,6 \pm 2,9$ & $18,1 \pm 3,2$ & $0,597 \quad$ \\
\hline 3 & $14,7 \pm 2,5$ & $14,2 \pm 2,6$ & $0,095 \quad "$ \\
\hline 4 & $21,8 \pm 2,4$ & $21,0 \pm 2,4$ & $0,289 \quad$ \\
\hline 5 & $21,8 \pm 3,4$ & $20,7 \pm 2,5$ & $0,378 \quad "$ \\
\hline $\begin{array}{l}\text { Injection } \\
\text { de }\end{array}$ & + & & \\
\hline 6 & $23,9 \pm 3,0$ & $19,4 \pm 3,9$ & $4,203 \quad "$ \\
\hline 7 & $10,2 \pm 3,5$ & $5,5 \pm 5,3$ & $2,885 \quad 1$ \\
\hline 8 & $10,2 \pm 2,5$ & $3,5 \pm 4,3$ & 10,526 HS \\
\hline 9 & $\begin{array}{l}\text { Injection de } \\
\text { vitamines } \\
17,5 \pm 4,9\end{array}$ & $\begin{array}{l}\text { Injection de } \\
\text { vitamines } \\
20,7 \pm 3,9\end{array}$ & $1,345 \mathrm{NS}$ \\
\hline 10 & $18,5 \pm 3,7$ & $15,9 \pm 4,2$ & $1,135 \quad "$ \\
\hline
\end{tabular}

\section{III.7. Esquisse économique}

Comme il a déjà été dit, le réélevage du yeau peut déboucher, dans les conditions locales sur 3 types de spéculations économiques : le veau de boucherie, le baby beef, le bœuf de labour ou le géniteur précoce.
A la lumière des résultats obtenus dans le lot 1 , on peut tenter de dégager les conditions économiques propres à chacune de ces spéculations.

Les éléments utilisés dans les calculs sont, d'une part, le prix de revient de l'UF alimentaire dans le lot 1 égal à $31 \mathrm{~F}$, d'autre part, les tendances générales dégagées dans les 3 graphiques suivants :

Les courbes présentées comportent 2 parties :
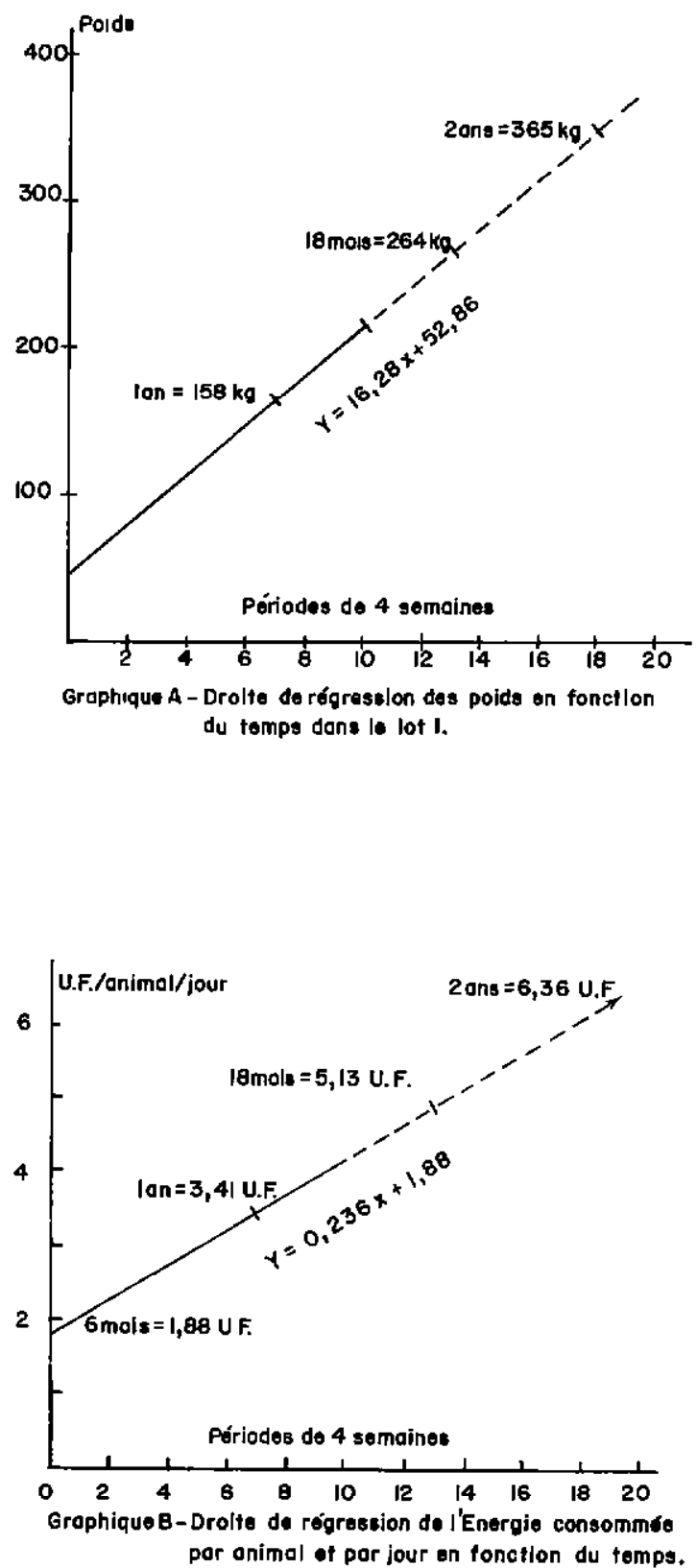


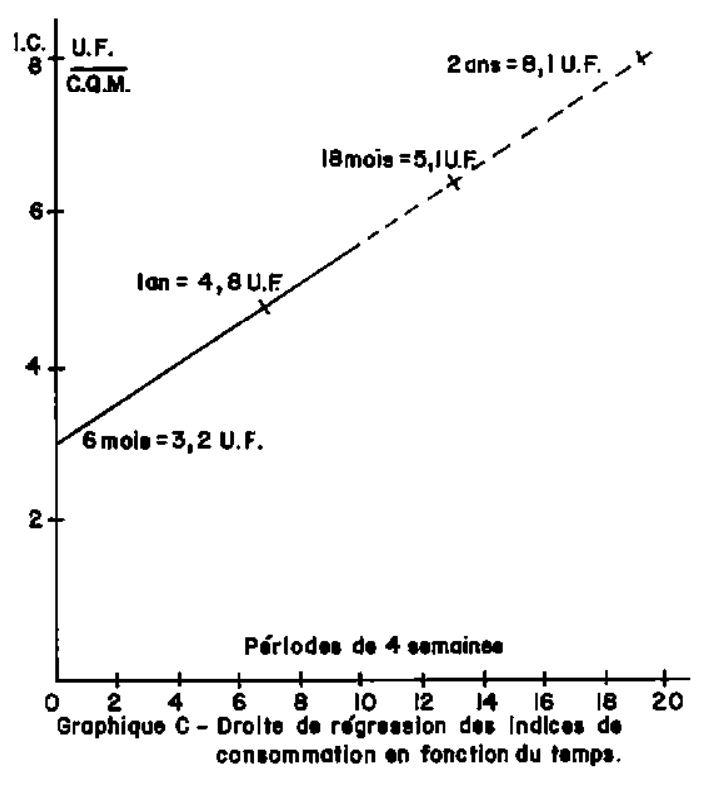

- une partie correspondant aux observations réelles, de 6 mois à 15 mois ;

- une partie extrapolée de 15 mois à 2 ans. Cette extrapolation nous paraît justifiable en ce qui concerne le graphique $A$, d'abord parce que dans la partie observée la liaison entre les poids et le temps est extrêmement étroite ( $r=0,995$ ) et, d'autre part, en raison d'une expérimentation antérieure sur le même type d'animaux qui avait montré une croissance de 6 mois à 2 ans parfaitement linéaire.

\section{III.7.1. Spéculation « veau de boucherie »}

Les veaux abattus au début août, dans le cadre de cette spéculation, pesaient en moyenne $150 \mathrm{~kg}$, ce qui d'après le graphique A correspond au poids obtenu après 6 mois d'une alimentation qui nécessite en moyenne par animal et par jour 2,65 UF.

Dans cette esquisse économique, on ne fait intervenir que les charges fixées, les plus importantes, représentées par l'achat des animaux, et le coût de leur nourriture.

- Achat $52 \times 65 \ldots \ldots=3380$

- Alimentation

$$
2,65 \times 31 \times 180 \ldots=14790
$$

TOTAL : ......... 18170 F CFA

Le prix de revient d'un $\mathrm{kg}$ de carcasse d'un poids moyen de $79,5 \mathrm{~kg}$ est donc de $228 \mathrm{~F}$. $\mathrm{Ce}$ prix est approximativement celui qu'on pouvait obtenir en août 1973, à l'abattoir de Dakar pour ce type de produit.
Donc avec 1 UF à $31 \mathrm{~F}$, la production du veau de boucherie après réélevage de 6 mois était donc, à l'époque, à peine rentable.

\section{III.7.2. Spéculation baby beef local}

Les animaux de ce type ont été commercialisés au poids vif de $290 \mathrm{~kg}$ qui correspond à l'âge de 20 mois si on s'en réfère au graphique $A$.

La période d'alimentation est donc ici beaucoup plus longue et les frais qu'elle entraîne beaucoup plus élevés.

Les charges fixes s'élèvent, dans ce cas à 51000 F CFA.

Les animaux abattus ont fourni des carcasses de $162 \mathrm{~kg}$. Le prix de revient du $\mathrm{kg}$ de carcasse s'élève done à $314 \mathrm{~F}$, prix nettement au-dessus de celui pratiqué à Dakar pour ce type d'animal. $\mathrm{Si}$ on admet à la vente un prix de $230 \mathrm{~F}$ le $\mathrm{kg}$, la commercialisation de ces animaux aurait produit 37260 et la spéculation aurait pu être équilibrée avec un aliment revenant à $22 \mathrm{~F}$ l'UF.

\section{III.7.3. Spéculation bauf de labour ou géniteur précoces}

Le graphique A montre qu'à 2 ans, avec ce type d'élevage, il est possible d'obtenir des animaux de plus de $350 \mathrm{~kg}$.

Les calculs effectués comme dans les cas précédents montrent que le coût de revient de ces animaux s'élève à $72350 \mathrm{~F}$, soit un prix de revient au $\mathrm{kg}$ vif de $200 \mathrm{~F}$, ce qui était absolument exclu.

Dans les conditions du marché en 73 et avec un prix de vente en vif de $100 \mathrm{~F}$ le $\mathrm{kg}$, ces animaux auraient pu être commercialisés pour $36000 \mathrm{~F} \mathrm{CFA}$. La spéculation aurait pu être possible si le prix de l'UF alimentaire avait été égal ou inférieur à $15 \mathrm{~F}$.

\section{DISCUSSIONS}

Cette expérimentation poursuivie sur de jeunes zébus de race Gobra et sur un échantillon restreint de métis Gobra $\times$ zébus pakistanais permet un certain nombre de réflexions intéressant les points suivants :

- performances obtenues et critères de croissance concernant l'élevage de ces types d'animaux ;

- influence du mode d'alimentation sur ces performances ; 
- rôle de la race dans les résultats obtenus, et évolution des données biochimiques ;

- économie de ce type de production.

\section{IV.1. Performances moyennes obtenues}

Les équations des droites de régression des poids en fonction du temps, présentées dans le tableau $n^{\circ}$ III, permettent de calculer le CQM obtenu dans chaque lot.

Dans le lot 1, il est de $540 \mathrm{~g}$. Pour apprécier le niveau de ce gain, nous pouvons invoquer un certain nombre d'éléments de comparaison.

Dans le milieu naturel, des pesées ont été effectuées au forage de Labgar, situé au centre de la zone sylvo-pastorale du Sénégal à l'occasion du projet FAC qui y a été récemment conduit. 195 " veaux " Gobra mâles, âgés de 2 ans «faits" ont accusé un poids moyen de $140 \mathrm{~kg}$ \pm 5 . Si on attribue à ces animaux un poids moyen à la naissance de $14 \mathrm{~kg}$, leur CQM au bout de 2 ans est de $170 \mathrm{~g}$.

A Dara, DENIS, VALENZA et THIONGANE, à propos de l'élevage extensif amélioré tel qu'il est conduit au C. R. Z., notent pour 227 individus âgés de 6 à 18 mois un CQM de $258 \mathrm{~g}$.

Au même centre, sont conduites depuis 1968 des expériences visant à l'extériorisation des potentialités génétiques du zébu Gobra par une alimentation optimale.

En 1968, pour 14 individus âgés de 6 à 18 mois, ces mêmes auteurs obtiennent un CQM de $698 \mathrm{~g}$ et, en 1970, sur 9 animaux et dans les mêmes conditions il n'est plus que de $515 \mathrm{~g}$.

Enfin, dans un contexte différent puisque se rapportant à l'Europe et à des races extrêmement précoces, on peut citer pour la race charolaise des CQM qui, entre la naissance et 14 mois, sont couramment supérieurs à $1100 \mathrm{~g} /$ jour.

La mesure de la consommation effectuée de façon précise dans les lots 1,2 et 3 durant les 6 premiers mois permet une approximation des besoins des jeunes zébus entre 6 et 12 mois. On peut tenter d'estimer durant cette période la part des besoins totaux revenant à l'entretien et la part nécessaire pour la croissance.

Pour calculer les besoins d'entretien, on utilise la formule déjà citée, faisant appel au poids métabolique :
B. entretien UF $=0,0364 \mathrm{P}^{0,75}$.

Dans ces conditions, les besoins de croissance (différence entre les besoins totaux et les besoins d'entretien) se sont élevés dans les lots 1,2 et 3 respectivement à $2,1-2,4$ et 2,5 UF pour $1 \mathrm{~kg}$ de gain.

Ces valeurs sont très comparables aux normes européennes telles que les cite CRAPLET, qui souligne en outre leur relative constance pendant une grande partie de la croissance. Cependant, cette constance ne se retrouve pas dans les conditions de l'expérience à Sangalkam. Les calculs précédents montrent, en effet, qu'il faut 1,8 UF pour produire $1 \mathrm{~kg}$ de gain à 6 mois, 2,9 UF à 12 mois et 4,7 UF à 18 mois. Cette augmentation rapide des besoins de croissance en fonction de l'âge pourrait tenir au fait que la ration utilisée n'a pas produit seulement de la croissance mais qu'il s'y est ajouté un certain engraissement qui, lui, est beaucoup plus coûteux.

Un autre facteur important de l'alimentation est constitué par l'apport azoté de la ration et son expression la plus efficace : le rapport protidique fourrager (MAD/UF).

L'animal jeune fait un croît proportionnellement plus riche en matières azotées que l'adulte qui dépose surtout de la graisse.

La ration du jeune doit donc tenir compte de ce fait et, chez lui, le rapport protidique fourrager doit être plus élevé.

Les normes dans ce domaine sont encore imprécises. Cependant, on admet que chez le veau de 1 à 2 mois, le rapport doit être voisin de 150. A 6 mois, il ne serait plus que de 135 pour passer aux environs de 100 à l'âge d'un an.

Entre 6 et 12 mois, dans le cadre de l'expérimentation et dans les lots 1,2 et 3, ce rapport s'est élevé respectivement à 135,125 et 123 .

Le coefficient d'encombrement (rapport matière sèche/unités fourragères) constitue encore une dernière notion importante. Dans la présente expérimentation et pour les premiers 6 mois, ce coefficient a varié de 1,8 dans le lot 1 à 1,0 dans le lot 3 en passant par 1,5 dans le lot 2.

Ces valeurs correspondent aux normes admises dans ce domaine. 
TABL. $N^{*} X I I I$ - Synthèse des rêsultats moyens dans chaque lot.

\begin{tabular}{|c|c|c|c|c|}
\hline & Lot 1 & Lot 2 & Lot 3 & Lot 4 \\
\hline C.Q.M. & $653 \pm 135$ & $568 \pm 115$ & $816 \pm 146$ & $486 \pm 115$ \\
\hline $\begin{array}{l}\text { M.5. consommée par } \\
100 \mathrm{~kg} \text { vif }\end{array}$ & $4,7 \pm 0,3$ & $3,9 \pm 0,3$ & $3,5 \pm 0,1$ & \\
\hline M.A.D./U.E. & 135 & 125 & 123 & \\
\hline $\begin{array}{l}\text { Indices de } \\
\text { consomation }\end{array}$ & $3,8 \pm 0,6$ & $4,5 \pm 0,4$ & $4,7 \pm 0,6$ & \\
\hline $\begin{array}{l}\text { Coefficient d'en- } \\
\text { combrement }\end{array}$ & 1,8 & 1,5 & 1,0 & \\
\hline
\end{tabular}

\section{IV.2. Comparaison des quatre lots durant les} six premiers mois

Le tableau XIII rappelle rapidement les résultats obtenus dans chaque lot.

Le gain de poids a été le meilleur dans le lot 3 composé de métis Gobra x Pakistanais venus du CRZ de Dara.

Ce gain est en effet très significativement différent de celui obtenu dans le lot $4(\mathrm{~F}=20,7)$ et dans le lot $2(F=11,8)$. Avec le lot $n^{\circ} 1$ la différence n'est pas significative $(F=4,4)$.

La comparaison de ce lot avec les 3 autres est cependant difficile car il est composé d'animaux à l'origine plus âgés et qui ont profité, à Dara, dans les premières périodes de leur vie d'une situation privilégiée par rapport aux autres.

Son avantage tient donc compte en partie des facteurs génétiques et en partie de meilleures conditions d'élevage réunies à Dara.

Parmi les Gobra, les meilleurs résultats ont été obtenus dans le lot 1 ce qui tient au type d'alimentation institué : mélange de coque d'arachide et de concentré dans ce cas, distribution séparée de fane d'arachide et de concentré dans les autres.

Enfin, le lot 4 a présenté la croissance la plus faible en raison de la pauvreté exceptionnelle du pâturage de saison sèche utilisé.

La consommation en matière sèche par $100 \mathrm{~kg}$ de poids vif a été très significativement plus élevée dans le lot 1 , ce qui s'est traduit évidemment par un coefficient d'encombrement supérieur.

Le lot 1 présente encore un indice de consommation significativement différent de celui des lots 2 et 3 . Les conditions alimentaires semblent donc meilleures que dans les autres lots.

\section{IV.3. Données biochimiques}

Le tableau $n^{\circ}$ XI présente l'évolution des données en fonction de la saison et de l'alimentation. Les modifications de faible amplitude qui interviennent en fonction de l'un ou l'autre facteur correspondent assez bien à celles déjà décrites et étudiées par FRIOT et CALVET dans l'article «Biochimie et élevage au Sénégal ». On y remarque en particulier, une fois de plus, que du point de vue des saisons, «le post-hivernage " semble correspondre à la saison où l'équilibre physiologique est le meilleur, non qu'on y rencontre les données les plus élevées mais celles qui se rapprochent le plus des normes telles que définies dans ce même article.

\section{IV.4. Considérations économiques}

Les discussions dans ce domaine tiennent compte du contexte économique existant en fin $73 \mathrm{car}$, depuis cette époque, le marché de la viande est devenu fluctuant et imprévisible.

Dans l'économie du réélevage tel qu'envisagé dans cette expérimentation, deux facteurs jouent un rôle prédominant: le prix de l'UF d'une part, le CQM obtenu de l'autre.

Nous rappelerons d'abord le prix de revient des aliments utilisés :

Concentré : $26 \mathrm{~F}$,

Fane d'arachide : $8 \mathrm{~F}$,

Prix UF du concentré : $31 \mathrm{~F}$.

Les veaux ont été achetés au prix de $65 \mathrm{~F}$ le $\mathrm{kg}$. Les prix de commercialisation en vif et en carcasses, tels qu'ils avaient été fixés par «un groupe de travail chargé de la fixation des prix de la viande », réuni en avril 1973, étaient les suivants :

$\begin{array}{cl}\text { - En vif : } 1^{\text {re }} \text { qualité } & : 110 \mathrm{~F} \mathrm{CFA,} \\ \text { animal tout venant } & : 70 \mathrm{~F} \mathrm{CFA,} \\ \text { maigre } & : 52,5 \mathrm{~F} \mathrm{CFA.}\end{array}$ 
- En carcasse : $1^{\text {re }}$ qualité : 215 F CFA, tout venant : $195 \mathrm{~F} \mathrm{CFA}$, maigre : 157 F CFA.

Nous allons d'abord, pour comparer l'économie des différents lots, utiliser une notion particulière, «le prix de vente de neutralisation » qui correspond au prix auquel il faudrait commercialiser le $\mathrm{kg}$ de vif pour équilibrer les «charges fixes " correspondant à l'achat des animaux et au coût de leur alimentation.

TABL. $\mathbb{N}^{\circ} X I V-P r i x$ de neutralisation des frais en cours de 1 'essai.

\begin{tabular}{|c|c|c|c|c|}
\hline Période & Lot 1 & Lot 2 & Lot 3 & Lot 4 \\
\hline 1 & 71 & 75 & 75 & 75 \\
2 & 76 & 81 & 79 & 77 \\
3 & 86 & 88 & 85 & 81 \\
4 & 92 & 93 & 89 & 84 \\
5 & 98 & 98 & 95 & 94 \\
6 & 102 & 101 & 100 & 97 \\
7 & 115 & & & 99 \\
8 & 129 & & & 99 \\
9 & 131 & & & 101 \\
10 & 136 & & & 105 \\
\hline
\end{tabular}

Pendant la saison sèche, les animaux des 4 lots se comportent, du point de vue économique, de façon équivalente.

Il n'en est plus de même dès l'apparition des pluies car, dans le lot 4, "les prix de neutralisations " se stabilisent autour de $100 \mathrm{~F}$ alors que dans le lot 1 , ils s'élèvent durant les mêmes périodes à $136 \mathrm{~F}$.

Il semble donc que, pour le réélevage et à condition de pouvoir disposer d'un pâturage convenable tout au long de l'année, la formule consistant à entretenir les animaux sur pâturage avec un supplément approprié, soit la meilleure.

Dans le cas où le pâturage ne peut remplir ces conditions tout au long de l'année, l'éleveur a cependant intérêt à mettre ses animaux au pâturage durant les 3 mois qui suivent le début des pluies.

Comme nous l'avons déjà signalé, dans les conditions expérimentales réalisées, seule la production du veau de boucherie était susceptible d'une rentabilisation. Au-delà de 6 mois, en effet, le prix de neutralisation des frais, comme le montre le tableau $n^{0}$ XIV, s'élève au-dessus du prix de vente qu'on peut obtenir.
Il faut donc tâcher, soit de diminuer le prix de revient de l'UF alimentaire, soit d'augmenter le niveau du CQM.

Nous avons calculé que, pour une rentabilisation de la spéculation baby beef, le prix de l'UF devait descendre au-dessous de $22 \mathrm{~F}$.

Avec le type de ration utilisée, ration sèche à base de sous-produits industriels, il était, en 1973, difficile de descendre à ce niveau. Une ration moins chère aurait pu cependant être obtenue en utilisant des sous-produits de l'industrie cotonnière (bourre de coton, coton graine et tourteau de coton), produits qui, dans des expérimentations d'embouche intensive antérieures se sont révélés comme étant d'une efficacité nutritive élevée. Mais des perspectives nouvelles semblent se dessiner avec la culture de plantes fourragères à haut rendement.

A Sangalkam, les essais de production de Panicum maximum, sous aspersion, permettent, en première approximation d'obtenir, en 1973, un prix de revient de l'UF voisin de $12 \mathrm{~F}$.

Les données de ce calcul sont les suivantes:

- $1 \mathrm{~m}^{3}$ d'eau donne $1,8 \mathrm{~kg}$ de matière sèche faisant 0,9 UF.

L'exhaure à partir de forage revient à $6 \mathrm{~F}$ le $\mathrm{m}^{3}$.

A l'eau s'ajoute le prix de l'engrais : $1,60 \mathrm{~F}$ par UF, les façons culturales: $0,50 \mathrm{~F} / \mathrm{UF}$ la coupe et la distribution : $3,5 \mathrm{~F} / \mathrm{UF}$.

L'économie du réélevage peut donc trouver un facteur très favorable dans ces perspectives nouvelles au Sénégal. A condition que ce type d'aliment donne d'aussi bons résultats que les rations sèches, la rentabilisation des 3 spéculations offertes au réélevage s'en trouverait assurée.

L'autre facteur susceptible d'intervenir dans l'économie de cette technique est le gain de poids journalier. Il est certain que si l'on augmente le CQM, la durée d'alimentation diminue et la rentabilisation augmente. Il ne semble pas cependant qu'on puisse envisager dans l'immédiat des perspectives nettement plus favorables dans ce domaine. Nous avons vu, en effet, que les expérimentations d'extériorisation conduites au CRZ de Dara dans le but, en particulier, de révéler les capacités de croissance maximale, avaient abouti à l'obtention d'un CQM comparable à celui obtenu à Sangalkam. Il est certain 
que, dans ce domaine, le facteur génétique joue un rôle prédominant.

Nous avons constaté l'effet favorable du métissage obtenu dans le lot 3 et il nous paraît que la poursuite de la sélection pratiquée à Dara ou bien le croisement avec des races très précoces, peuvent seuls parvenir à élever sensiblement le CQM au-dessus du niveau obtenu à Dara ou à Sangalkam.

Cette affirmation semble en contradiction avec les résultats obtenus au cours des essais d'embouche intensive pratiqués à Dakar avec des rations de même type. En effet, des CQM supérieurs à $800 \mathrm{~g}$ ont été couramment obtenus au cours de période de 3 ou 4 mois et sur des animaux âgés en moyenne de 4 ans. C'est qu'il ne faut pas oublier que, dans ces derniers cas, est intervenu un phénomène très important, celui de la "croissance compensatrice » dont l'efficacité est capable d'expliquer les différences notées.

Chez les jeunes tels qu'ils se présentent au cours de cette expérimentation, la croissance compensatrice joue très peu et, si elle a été susceptible d'influencer légèrement les données de départ, son efficacité disparaît au fur et à mesure que se prolonge la période d'alimentation.

\section{v. CONCLUSIONS}

L'élevage du veau hors de son milieu de naissance constitue une méthode d'accélération rapide de la production. Dans l'expérimentation réalisée à Sangalkam, le poids des veaux est passé de $50 \mathrm{~kg}$ à l'âge approximatif de 6 mois à $160 \mathrm{~kg}$ à 1 an et on aurait pu escompter un poids de plus de $360 \mathrm{~kg}$ à 2 ans. Il y a donc un raccourcissement considérable de la production.
Cependant, cette spéculation, capable de déboucher sur la production d'un veau de boucherie local, d'un baby beef adapté aux conditions africaines ou d'un animal de labour précoce dont l'agriculture a un grand besoin, bute très rapidement sur des problèmes économiques.

Ceci tient essentiellement à deux facteurs : - un prix de l'UF élevé dans les conditions expérimentales décrites ;

- un CQM plus bas que celui qu'on pouvait escompter à la lumière des essais d'embouche intensive sur taurillon.

A ces facteurs limitants de cette production, deux remèdes peuvent être proposés :

- I'utilisation de plantes fourragères ou d'ensilage susceptibles de diminuer sensiblement le prix de revient de l'UF ;

- les croisements industriels capables d'entraîner un sensible relèvement du gain quotidien moyen.

Les futures expérimentations doivent s`inspirer de ces deux considérations.

Nous n'avons évidemment envisagé dans cet article que l'aspect technique du problème. L'incitation, la participation et, en dernière analyse, le bénéfice des éleveurs, demeurent les réels moteurs de cette révolution dans les techniques de l'élevage sahélien tel qu'il est pratiqué depuis des temps très anciens.

\section{REMERCIEMENTS}

Nous avons apprécié l'assistance technique et la collaboration de MM. DIALLO, FRIOT, PAGE LECUYER, VALENZA, des services de Chimie, Physiologie, Agrostologie; de M. WANE et du personnel de la Ferme annexe de Sangalkam. Qu'ils trouvent ici l'expression de nos sincères remerciements.

\section{SUMMARY}

\section{Prospects on calves rebreeding in Senegal and sahelian areas}

After several years of severe drought, the restoration of sahelian cattle herds must be directed to a more intensive management.

With this purpose, among various solutions, one consists in an early calves withdrawal from sahelian zone (birth areas) to places where ecological and feeding conditions are more suitable. There young animals would quickly reach their slaughtering weight.

According to this scheme, an experiment was carried out in Sangalkam farm, during nine month. Economical and technical results are reported and discussed by the authors. 


\section{RESUMEN}

Perspectivas concerniendo a la recria de los terneros en Senegal y en zona saheliana

Después de los ultımos años de sequedad, la reconstitución de la ganaderia saheliana necesitaria una orientación hacia un modo de explotación más intensivo. Una de las soluciones seria la salida temprana de los terneros hacia regiones donde las condiciones ecologicas y alimenticias son mejores. Ahi, los jovenes animales podrian llegar a su peso de matanza.

Se ha experimentado un ensayo de este tipo durante 9 meses utilizando varios modos de alımentación en Sangalkam, una granja situada al norte de Dakar.

Los autores presentan y discuten los resultados técnicos y económicos.

\section{BIBLIOGRAPHIE}

CRAPLET (C.). Traité d'élevage moderne. Les Bovins, tomes III et VIII, Paris, Vigot Frères.

DENIS (J. P.), VALENZA (J.). Extériorisation des potentialités du Zébu Gobra. Rev. Elev. Méd. vét. Pays trop., 1971, 24 (3) : 409-418.

DENIS (J. P.), VALENZA (J.) et THIONGANE (A. I.). Extériorisation des potentialités du Zébu Gobra. Résultats d'abattages. Rev. Elev. Méd. vét. Pays trop., 1972,25 (2) : 245-257; 1974, 27 (1) : 109-114.

DUKES (H. H.). The physiology of domestic animals. $7^{\mathrm{e}}$ éd., Ithaca, New York, Comstock publ. Ass., 1964.

FRIOT (D.), CALVET (H.). Biochimie et élevage au Sénégal. $8^{\mathrm{e}}$ Journées Médicales de Dakar, 9-14 avril 1973.

JACQUOT (R.) et collab. Physiologie des animaux domestiques. Paris, Vigot Frères, 1964.

RIVIÊRE (R.). Introduction générale; pp. 7 à 9. Synthèse et conclusion: pp. 323 à 330 . L'embouche bovine en Afrique tropicale et à Madagascar, Colloque de Dakar: 4-8 décembre 1973. 